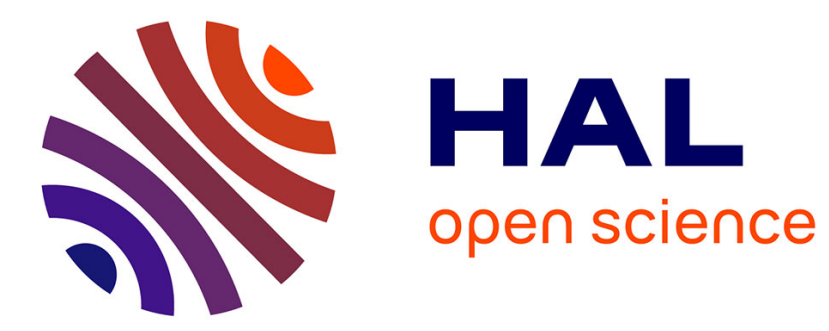

\title{
Etude des verres du système Ag-Ge-Te
}

\author{
A. Ferhat, R. Ollitrault-Fichet, V. Mastelaro, S. Bénazeth, J. Rivet
}

\section{To cite this version:}

A. Ferhat, R. Ollitrault-Fichet, V. Mastelaro, S. Bénazeth, J. Rivet. Etude des verres du système AgGe-Te. Journal de Physique IV Proceedings, 1992, 02 (C2), pp.C2-201-C2-206. 10.1051/jp4:1992229 . jpa-00251300

\section{HAL Id: jpa-00251300 https://hal.science/jpa-00251300}

Submitted on 1 Jan 1992

HAL is a multi-disciplinary open access archive for the deposit and dissemination of scientific research documents, whether they are published or not. The documents may come from teaching and research institutions in France or abroad, or from public or private research centers.
L'archive ouverte pluridisciplinaire HAL, est destinée au dépôt et à la diffusion de documents scientifiques de niveau recherche, publiés ou non, émanant des établissements d'enseignement et de recherche français ou étrangers, des laboratoires publics ou privés. 


\title{
Etude des verres du système Ag-Ge-Te
}

\author{
A. FERHAT ${ }^{*}$, R. OLLITRAULT-FICHET ${ }^{*}$, V. MASTELARO ${ }^{* * *}$, S. BÉNAZETH ${ }^{*, * *}$ et J. RIVET \\ "Laboratoire de Chimie Minérale Structurale, (CNRS U.R.A. 200), Faculté des Sciences Pharmaceutiques \\ et Biologiques, 4 avenue de l'Observatoire, 75270 Paris Cedex 06, France \\ ${ }^{* *}$ LURE, Université Paris-Sud, 91405 Orsay, France
}

A middle-sized vitreous domain resulting from binary glasses of the Ge-Te system has been found. DTA measurements of the glass-transition ( $\mathrm{Tg}$ ) and crystallization $(\mathrm{Tc})$ temperatures have been performed which show that, for a constant value of the $\mathrm{Ge} / \mathrm{Te}$ ratio, the values of these parameters do not depend on the silver content. Electrical properties of the vitreous $\mathrm{Ag}_{\mathrm{x}} \mathrm{GeTe}_{4.7}$ system (with $0.00 \leq x \leq 1.40$ ) have been investigated by means of the complex impedance method. At $25^{\circ} \mathrm{C}$, electrical conductivity and activation energies vary within $10^{-5}$ and $10^{-4} \Omega^{-1} \cdot \mathrm{cm}^{-1}$ and within 0.47 and $0.42 \mathrm{eV}$, respectively. Optical gap and e.m.f. measurements as well as the determination of the electronic transference number by means of electrochemical semipermeability flux measurements reveal a semiconductor behaviour in spite of the rather high silver content. In order to investigate the local structure around the silver and germanium atoms, an EXAFS structural approach has been undertaken. The obtained results show that in ternary glasses the arrangement of the tellurium atoms around a germanium atom is appreciably the same as that found in binary glasses. It can be noticed that Fourier transform moduli are higher for glassy phases than for crystalline ones; such a feature has previously been observed in the As-Te system. Besides, $\mathrm{Ag}^{+}$ions appear to be tetracoordinated.

Le diagramme de phases du système Ag-Ge-Te [1] a été étudié par analyse thermique différentielle, diffraction de rayons $X$ et analyse métallographique. Le tracé des vallées eutectiques du diagramme de phases, représenté sur la figure 1, montre :

- l'existence d'un composé ternaire à décomposition péritectique de type binaire,

- l'apparition d'une importante zone de démixtion ternaire à deux liquides s'appuyant sur la démixtion du binaire $\mathrm{Ag}-\mathrm{Ag}_{2} \mathrm{Te}$ et presque complètement localisée dans le quadrilatère $\mathrm{Ag}-\mathrm{Ag}_{2} \mathrm{Te}-\mathrm{Ag}_{8} \mathrm{GeTe}_{6}$,

- la présence de sept invariants ternaires : quatre eutexies et trois péritexies transitoires,

- et enfin le développement d'une zone de verre de moyenne étendue, issue de celle du binaire Ge-Te (comprise entre 13 et 22 at. p. cent de Ge). Elle chemine le long d'une vallée eutectique. La teneur maximale en argent à l'intérieur du domaine vitreux est de 23 p. cent. C'est l'étude de ces verres qui fait l'objet de ce travail. 
Les échantillons vitreux ont été obtenus par trempe, dans l'eau froide, depuis la température de $900^{\circ} \mathrm{C}$. Certaines compositions situées le long de la vallée eutectique peuvent être obtenues à l'état vitreux par suite de refroidissement lent $\left(5^{\circ} \mathrm{C} . \mathrm{mn}^{-1}\right)$ depuis l'état liquide.

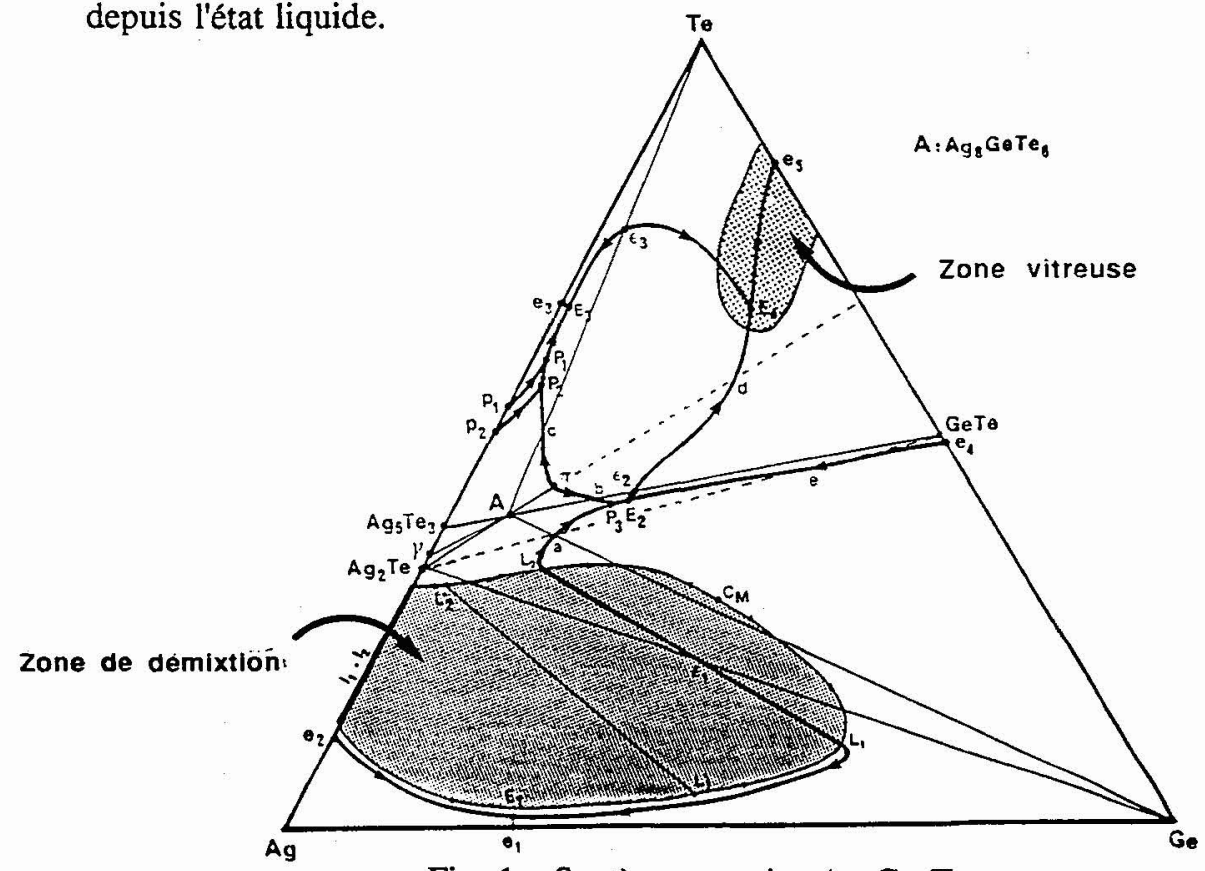

Fig. 1 - Système ternaire Ag-Ge-Te

Les températures de transition vitreuse $\mathrm{Tg}$ et les températures de cristallisation Tc (Fig. 2 et 3 ) sont obtenues à partir des thermogrammes.

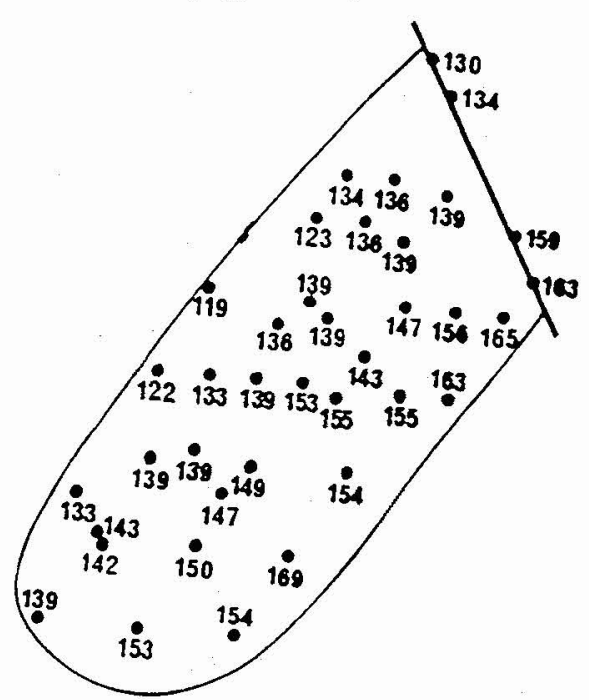

Fig. 2 - Températures de transition vitreuse $(\mathrm{Tg})$

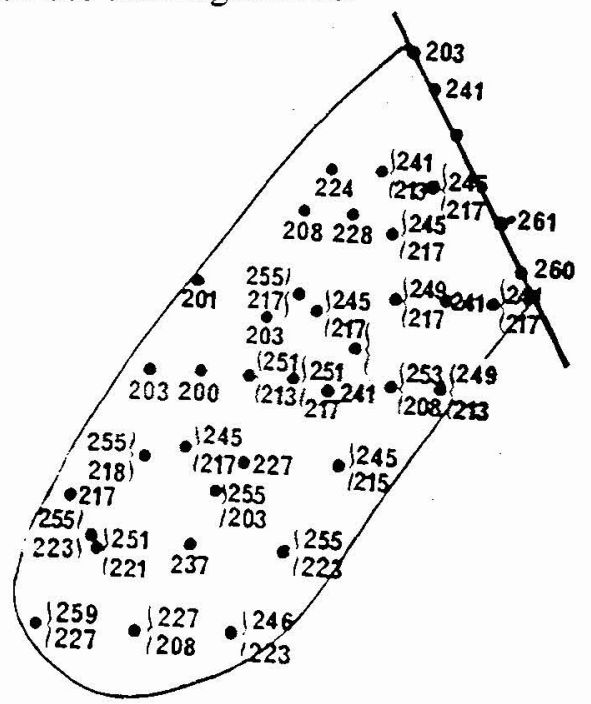

Fig. 3 - Températures de cristallisation (Tc) 
Pour une teneur constante en tellure, les températures de transition vitreuse $\mathrm{Tg}$ diminuent légèrement quand la teneur en argent augmente. Par ailleurs, ces mêmes températures ne varient pas en fonction de la teneur en argent lorsque le rapport $\mathrm{Ge} / \mathrm{Te}$ demeure constant. Ces comportements montrent que les verres ternaires conservent la structure des verres binaires à base de germanium et de tellure. Le réseau covalent des verres binaires se retrouvent sans grande modification dans les verres ternaires. Cette constatation est confirmée dans l'approche structurale par spectroscopie EXAFS.

Une étude des propriétés électriques des verres $\mathrm{Ag}-\mathrm{Ge}-\mathrm{Te}$ par la méthode des impédances complexes, pour les verres de composition $\mathrm{Ag}_{\mathrm{x}} \mathrm{GeTe}_{4,7}$ (avec $\mathrm{x}=0,00 ; 0,10$; $0,25 ; 0,50 ; 1,00 ; 1,40)$ a été entreprise. Les mesures ont été effectuées entre 25 et $80^{\circ} \mathrm{C}$. Les courbes de la conductivité en fonction de l'inverse de la température absolue sont des droites qui traduisent un comportement semi-conducteur. A $25^{\circ} \mathrm{C}$ quand le pourcentage d'argent crôit de 0 à $20 \mathrm{p}$. cent, la conductivité électrique augmente environ de $10^{-5}$ à $10^{-4} \Omega^{-1} \cdot \mathrm{cm}^{-1}$. Parallèlement, les énergies d'activation décroissent légèrement de 0,47 à $0,42 \mathrm{eV}$ (Fig. 4).

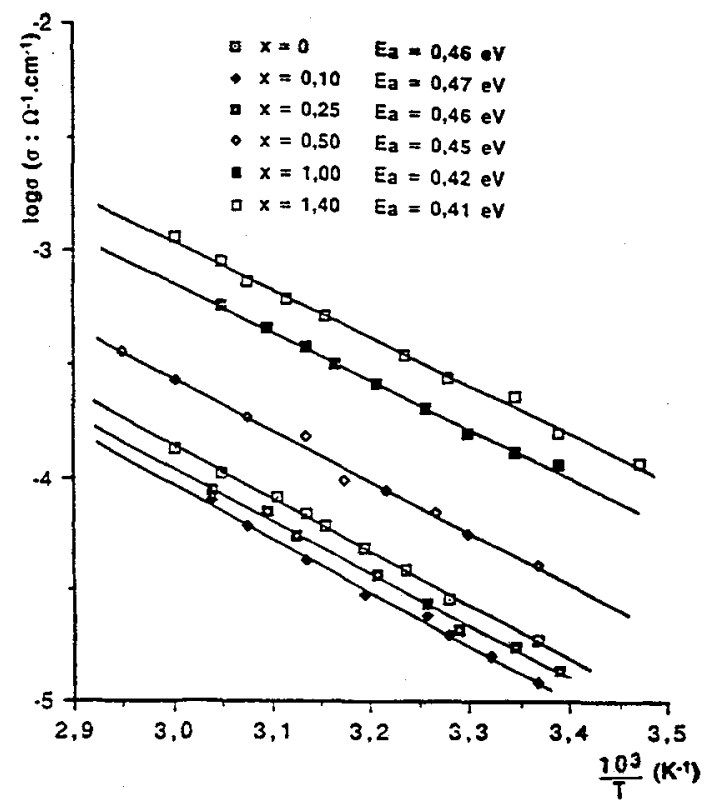

Fig. 4 - Conductivité des verres de composition $\mathrm{Ag}_{\mathrm{x}} \mathrm{GeTe}_{4,7}$ en fonction de $1 / \mathrm{T}$

Pour les différentes teneurs en argent correspondant aux échantillons étudiés, le diagramme des impédances complexes ne laisse apparaître aucun phénomène d'électrode à basse fréquence, ce qui tendrait à prouver que le transport de charge n'est pas assuré par les ions $\mathrm{Ag}^{+}$. Des mesures de gap optique ont été entreprises. Les valeurs obtenues sont environ égales au double de celles correspondant à l'énergie d'activation, ce qui tend encore à confirmer le caractère semi-conducteur de ces verres. Le nombre de transport ionique de ces verres a été déterminé par la méthode des f.é.m.. A $25^{\circ} \mathrm{C}$, quelque soit le pourcentage d'argent des échantillons étudiés, les verres se caractérisent par des f.é.m. très faibles de l'ordre de $10^{-3} \mathrm{~V}$, ce qui correspond à un nombre de transport ionique négligeable.

Afin de confirmer les résultats précédents, nous avons fait une estimation du nombre de transport électronique par la méthode dite de la "semi-perméabilité électronique". Cette méthode consiste à interposer l'échantillon vitreux entre deux solutions à base de mercure, l'une saturée en argent, l'autre en contenant seulement des traces. On suit l'évolution du potentiel de la cellule en fonction du temps. Pour les verres examinés, on observe une décroissance rapide du potentiel qui s'annule au bout de quelques heures. Ce résultat est caractéristique d'une conduction électronique appréciable, justifiant ainsi les résultats précédents. Le recuit des verres, à une température légèrement supérieure à $T g$, entraîne une nette croissance de la conduc- 
tivité et une diminution de l'énergie d'activation (Fig. 5). Ce comportement est dû à la formation d'une phase ternaire et à l'apparition de tellure au cours du recuit_.

Après avoir étudié les propriétés chimiques et physiques de ces verres, nous avons entrepris une approche structurale par spectroscopie EXAFS dans le but de mettre en évidence des relations structurepropriétés. Nous avons donc étudié l'environnement du germanium pour tenter d'établir si les motifs structuraux des verres binaires sont bien identiques à ceux des verres ternaires. Par ailleurs, nous avons également étudié l'environnement du cation $\mathrm{Ag}^{+}$afin de préciser son mode de liaison avec les atomes de tellure, la conductivité étant, ici, de nature exclusivement électronique. Cette étude de l'environnement du cation $\mathrm{Ag}^{+}$est pour nous particulièrement intéressante étant donné que, dans notre laboratoire, nous disposons des résultats de systèmes où la conductivité est au contraire essentiellement ionique (Ag-As-S et Ag-As-Se) [2].

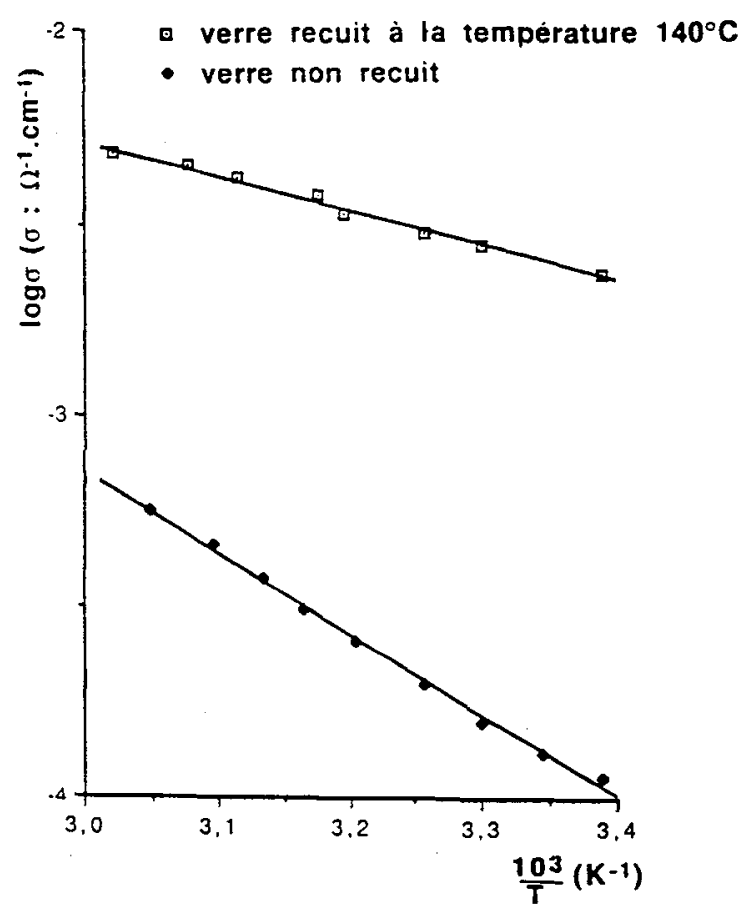

Fig. 5 - Conductivité du verre de composition $\mathrm{AgGeTe}_{4,7}$ recuit à $140^{\circ} \mathrm{C}$ en fonction de $1 / \mathrm{T}$

Nous pensons qu'une étude comparative des modes de liaisons de l'argent dans des matrices vitreuses sulfurées, séléniées ou tellurées pourrait être riche d'information, le tellure étant connu pour présenter un caractère métallique plus prononcé. Il ne nous a pas été possible, pour des raisons liées à la technique ellemême, d'enregistrer des spectres au seuil $\mathrm{K}$ du tellure.

En ce qui concerne l'environnement du germanium, la comparaison des spectres EXAFS normalisés montre une très bonne similitude entre la phase cristalline et les compositions vitreuses binaire ou ternaire. Ce comportement laisse prévoir que les environnements sont voisins et justifie l'utilisation des valeurs des phases et amplitudes, pour la paire Ge-Te, extraites de cette référence cristalline. Cependant la comparaison des modules des tansformées de Fourier des spectres normalisés (Figure 6) montre que l'amplitude du pic caractéristique des premiers voisins du germanium est plus intense dans la phase cristalline que dans les deux verres. Nous verrons que cet état s'interpréte par un état de désordre plus grand dans les verres. Les détails du traitement des données expérimentales seront reportés dans une publication ultérieure, nous nous limitons ici à une analyse des paramètres structuraux.

Après affinement de ces paramètres (Tableau 1) on obtient une remarquable 
superposition des spectres expérimentaux et calculés.

En ce qui concerne le verre binaire aucune étude de l'environnement du germanium par spectroscopie d'absorption n'est à ce jour décrite dans la littérature, nos résultats doivent donc être comparés à ceux obtenus par d'autres techniques [3, 4]. Les auteurs proposent des modèles 3-3 ou 4-2 pour les coordinations de Ge et de Te. Notre étude permet d'exclure l'hypothèse d'une coordination 3-3. Nous ne mettons pas en évidence de liaisons homopolaires Ge-Ge pour cette composition vitreuse, c'est-à-dire que l'ordre chimique est bien respecté dans les verres de germanium alors que nous avons montré, en associant à l'EXAFS des expériences de diffusion anomale de rayons $X$, que cela n'était pas le cas des verres binaires As-Te [5, 6]. Il faut noter ici que le domaine de formation vitreuse des verres binaires Ge-Te est limité aux compositions riches en tellure et permet, à priori, de ne pas d'émettre l'hypothèse de l'existence de liaisons Ge-Ge. Afin de mieux appréhender, sur de plus longues distances, la structure de ces verres binaires Ge-Te, nous allons entreprendre dans ce cas aussi, une étude par diffusion anomale de rayons $\mathrm{X}$ au LURE.

Sur le tableau 1 , nous remarquons que les coordinations des atomes de germanium sont identiques pour les deux verres binaire et ternaire. Cette ressemblance structurale est en bon accord avec la similitude des résultats obtenus lors des études thermiques et électriques.

\begin{tabular}{|c|c|c|c|c|}
\hline $\begin{array}{c}\text { Compositions } \\
\text { des verres }\end{array}$ & $N$ & $R(\AA)$ & $\Delta \sigma(\AA)$ & $\Delta E(\mathrm{eV})$ \\
\hline $\mathrm{GeT}_{\mathrm{e}_{4}}$ & 3,90 & 2,60 & 0,04 & $-7,847$ \\
$\mathrm{Ag}_{7} \mathrm{Ge}_{18} \mathrm{~T}_{75}$ & 4,00 & 2,59 & 0,04 & $-4,767$ \\
\hline
\end{tabular}

Tableau 1 Ajustement à une couche au seuil $\mathrm{K}$ du germanium

$$
\begin{aligned}
& \mathrm{N} \quad \text { : nombre de voisins de tellure autour du germanium } \\
& \mathrm{R} \quad: \text { distance moyenne entre le germanium et le teliure } \\
& \Delta \sigma \quad: \text { écart entre le facteur Debye-Waller de la référence } \\
& \text { cristalline et celui du verre }
\end{aligned}
$$

Nous abordons maintenant l'étude de l'environnement de l'argent. Les modules des transformées de Fourier sont représentés sur la figure 7.

En ce qui concerne les spectres EXAFS au seuil K $(25520 \mathrm{eV})$ de l'argent nous disposions de la même composition vitreuse ternaire et de la phase cristallisée $\mathrm{Ag}_{5} \mathrm{Te}_{3}$. Dans cette dernière, l'argent présente un environnement tétraédrique quasirégulier $[R(\mathrm{Ag}-\mathrm{Te})=2,98 \AA ̊ \AA$. Ce composé a donc pu servir de référence lors de l'analyse des données. Les affinements des paramètres structuraux nous montrent, sans ambiguïté, que ce verre ternaire présente une coordination tétraédrique autour de l'argent avec une distance voisine de celle du cristal $\left[R_{(A g-T e)}=2,94 \AA\right]$. La plus faible valeur de l'amplitude du module de la transformée de Fourier du cristal (Fig. 7), comparée à celle du verre s'explique par le fait que dans la phase cristalline $\mathrm{Ag}_{5} \mathrm{Te}_{3}$ il existe un plus grand désordre structural : seulement $82 \%$ des atomes d'argent sont dans des sites tétraédriques, les $18 \%$ restant ont une coordinence triangulaire ou linéaire [7]. Cet environnement de l'argent est spécifique des verres tellurés et nous n'avons pas trouvé une telle valeur lorsque nous avons étudié les 
verres des systèmes ternaires Ag-As-S et Ag-As-Se [2]. En effet, dans de tels systèmes, l'argent est dicoordiné ; ce fait pourrait caractériser sa conduction ionique. Il est facile d'admettre alors que si les ions $\mathrm{Ag}^{+}$sont engagés dans des coordinations d'ordre 4 , ils ne trouvent plus la mobilité nécessaire pour assurer un mode de conduction ionique.

En conclusion il est intéressant de comparer les propriétés physico-chimiques et structurales de ces verres tellurés avec celles des verres séléniés. Ces derniers présentent une conductivité ionique non négligeables [8]. Alors que malgré leur teneur importante en argent, les verres tellurés présentent un caractère semiconducteur. L'étude structurale permet de situer les cations $\mathrm{Ag}^{+}$dans des tétraèdres tellurés, ce qui explique leur diminution de mobilité.

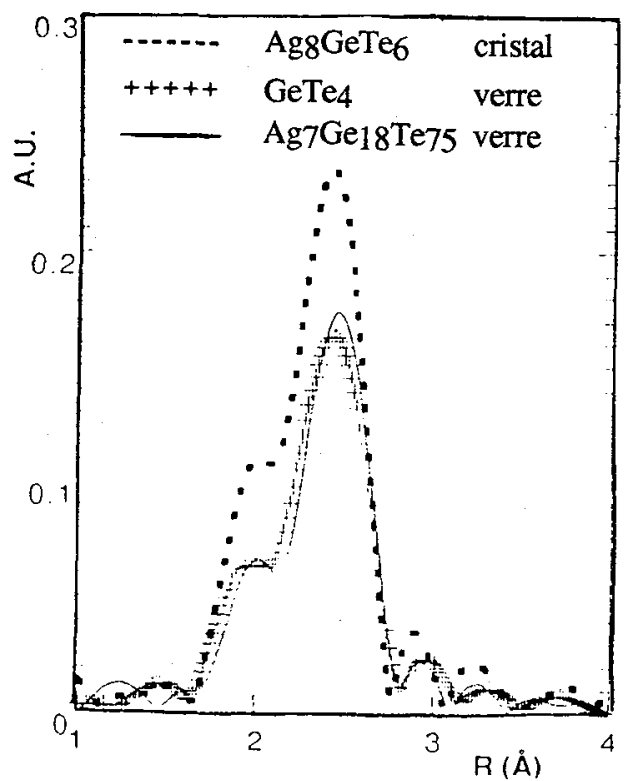

Fig. 6. Modules des transformées de Fourier des signaux d'EXAFS au seuil $\mathrm{K}$ du germanium

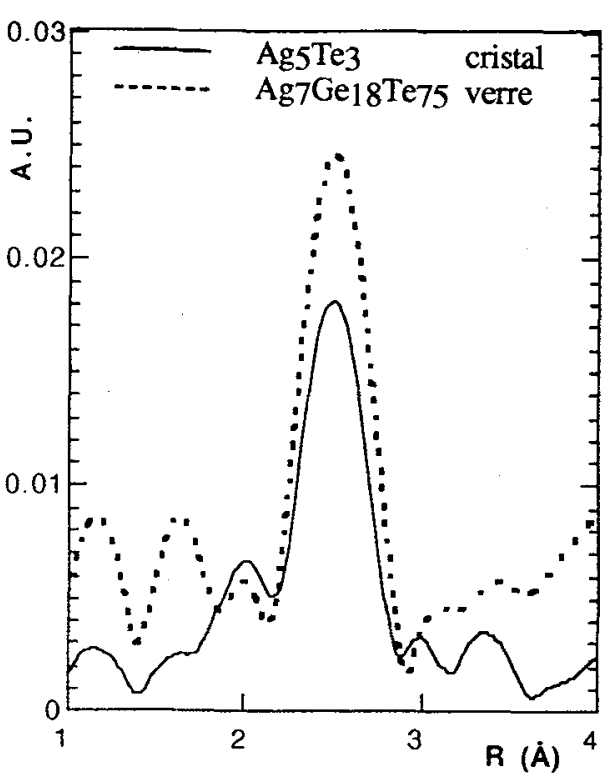

Fig.7 - Modules des transformées de Fourier des signaux d'EXAFS au seuil $\mathrm{K}$ de l'argent

[1] FERHAT A.. OLLITRAULT-FICHET R. and RIVET J. J. Alloy and Compounds 177 (1991) 337.

[2] MASTELARO V., BÉNAZETH S., OLLITRAULT-FICHET R., IBANEZ A., DEXPERT H. and DUGUÉ J.

Conference Proceeding, Vol 25 "2nd European Conference on Progress in X-Ray Synchrotron Radiation Research" A. Balerna, E. Bernieri and S. Mobilio (Eds.), SIF, Bologna (1990).

[3] BETTS F., BIENENSTOCK A. and OVSHINGSKY S. R.

J. Non Cryst. Solids, 4 (1970) 554.

[4] BETTS F., BIENENSTOCK A., KEATING D. T. and NEUFVILLE J. P. J. Non Cryst. Solids, 7 (1972) 417.

[5] MA Q., BENAZETH S. and RAOUX D.

X-Ray absorption fine structure (1991).S. Hasnain ed. 715.

[6] MA Q., BENAZETH S. and RAOUX D.

Proc. int. Conf. of Non Cryst. Solids Cambridge, To be published.

[7] JAULMES S., JULIEN-POUZOL M., DUGUÉ J. et RIVET J.

Acta Cryst. (à paraître).

[8] KOBLENCS M.Thèse de 3ème cycle. Université Pierre et Marie Curie. Paris (1983). 